PROCEEDINGS OF THE

AMERICAN MATHEMATICAL SOCIETY

Volume 130, Number 8, Pages 2305-2309

S 0002-9939(02)06505-X

Article electronically published on March 8, 2002

\title{
CONTINUOUS TRANSFORMATION OF BAIRE MEASURES INTO LEBESGUE MEASURE
}

\author{
HANS G. KELLERER
}

(Communicated by David Preiss)

\begin{abstract}
A recent result by Wulbert on the existence of continuous functions with measure zero level sets is slightly extended and its proof is considerably simplified. As a by-product, a criterion is established for a Baire measure to allow a continuous transformation into Lebesgue measure.
\end{abstract}

\section{INTRODUCTION}

As outlined in the survey [5] by Wulbert, the following result is of interest in $L_{1}$-approximation theory:

(*) Given a nonatomic $\sigma$-finite measure space $(X, \mathfrak{A}, \mu)$, for any finite dimensional subspace $\mathcal{K}$ of $\mathcal{L}_{1}(X, \mathfrak{A}, \mu)$ there is an extreme element $g$ in the unit ball of the dual space, i.e. satisfying

$$
g(x)= \pm 1 \quad \text { for } \mu \text {-almost all } x \in X,
$$

which annihilates $\mathcal{K}$, i.e. satisfies

$$
\int_{X} f g d \mu=0 \quad \text { for all } f \in \mathcal{K} .
$$

One approach to this result uses Liapunov's convexity theorem, as has been carried out by Phelps and Dye [4 Theorem 2.5]. An alternative, yielding some additional insight, consists in applying the Borsuk-Ulam theorem on antipodal mappings, as has been done by Hobby and Rice [1] in the classical case $X=[0,1]$ and $\mu=\lambda(:=$ Lebesgue measure). In fact, this approach has been anticipated in a stochastic context by the present author [2, Satz 3] for arbitrary measure spaces. The additional tool there is a transformation of the underlying measure $\mu$ into Lebesgue measure, as is always available under the - obviously necessary - condition that $\mu$ is nonatomic and $\sigma$-finite. Actually, all that is needed is an $\mathfrak{A}-$ measurable function $h$ with measure zero level sets, i.e.

$$
\mu(\{x: h(x)=y\})=0 \quad \text { for all } y \in \mathbf{R} .
$$

In a recent paper [6] Wulbert proves that in the topological situation the function $h$ may even be chosen to be continuous, which yields for $(*)$ above a solution $g$ being the sign of a continuous function. Since his construction is highly involved, a simple proof seemed to be desirable. On the way it turned out that by a slight generalization the question raised by the title of this paper finds a complete answer.

Received by the editors January 5, 2001

2000 Mathematics Subject Classification. Primary 28C15; Secondary 46 G10.

Key words and phrases. Baire measures, measure transformation, Lebesgue measure. 


\section{PRELIMINARIES}

In proving the main results, three simple lemmata will be used. First, the notion of moderatedness has to be extended from Borel to Baire measures:

Definition 1. For an arbitrary topological space $X$ denote by $\mathfrak{C}(X)$ the Baire $\sigma$-algebra, generated by the space $\mathcal{C}(X)$ of continuous functions. Then a measure $\mu$ on $\mathfrak{C}(X)$ is called "moderated", if $\mathcal{C}(X)$ contains functions $f_{n}, n \in \mathbf{N}$, satisfying

$$
X=\bigcup_{n \in \mathbf{N}}\left\{x: f_{n}(x) \neq 0\right\} \quad \text { and } \quad \mu\left(\left\{x: f_{n}(x) \neq 0\right\}\right)<\infty \text { for all } n \in \mathbf{N} .
$$

While any moderated Baire measure is $\sigma$-finite, the converse fails even for nonatomic measures. Indeed, choose an open base $G_{n}, n \in \mathbf{N}$, for $X=[0,1]$ and measurable functions $f_{n} \geq 0, n \in \mathbf{N}$, such that

$$
\int_{G_{n}} f_{n} d \lambda=\infty \quad \text { and } \quad \sum_{n \in \mathbf{N}} \lambda\left(\left\{x: f_{n}(x) \neq 0\right\}\right)<\infty .
$$

The last condition implies

$$
\lambda\left(\left\{x: f_{n}(x)=0 \text { for almost all } n \in \mathbf{N}\right\}\right)=1,
$$

hence $f:=\sum_{n \in \mathbf{N}} f_{n}$ is $\lambda$-almost finite and thus $d \mu=f d \lambda$ defines a $\sigma$-finite measure. Due to $\mu(G)=\infty$ for all open sets $G \neq \emptyset$, however, it fails to be moderated (this example also solves the final problem in [6]).

The following criterion will be required in the sequel:

Lemma 1. A Baire measure $\mu \mid \mathfrak{C}(X)$ is moderated if and only if $\mathcal{C}(X)$ contains a $\mu$-integrable strictly positive function $f$.

Proof. Given functions $f_{n}, n \in \mathbf{N}$, according to Definition 1 , choose constants $\gamma_{n}>0$ such that

$$
\sum_{n \in \mathbf{N}} \gamma_{n}<\infty \quad \text { and } \quad \sum_{n \in \mathbf{N}} \gamma_{n} \mu\left(\left\{x: f_{n}(x) \neq 0\right\}\right)<\infty .
$$

Then the function

$$
f:=\sum_{n \in \mathbf{N}}\left(\left|f_{n}\right| \wedge \gamma_{n}\right)
$$

meets all requirements. Conversely, if $f$ is strictly positive and $\mu$-integrable, then the functions

$$
f_{n}:=\left(f-\frac{1}{n}\right)^{+}, n \in \mathbf{N},
$$

satisfy the conditions of Definition 1.

Next, some notation has to be introduced:

Definition 2. For a Hausdorff topological space $Y$ denote by $\mathfrak{B}(Y)$ the Borel $\sigma$-algebra and by $M(Y)$ the space of all finite measures $\nu$ on $\mathfrak{B}(Y)$ endowed with the weak (narrow) topology. Then

$$
\delta(\nu):=\max _{y \in Y} \nu(\{y\}) \quad \text { for } \nu \in M(Y) .
$$

The following fact will be used only for $Y=\mathbf{R}$, but is of independent interest:

Lemma 2. If $Y$ is a second countable metrizable space, then the functional $\delta$ is upper semicontinuous. 
Proof. Fix $\nu_{0} \in M(Y)$ and $\delta_{0}>0$ with $\delta\left(\nu_{0}\right)<\delta_{0}$. Since $Y$ is second countable, $\nu_{0}$-atoms are singletons, which is easily seen to imply that $Y$ may be partitioned into a finite number of sets $B_{m} \in \mathfrak{B}(Y), 1 \leq m \leq n$, with $\nu_{0}\left(B_{m}\right)<\delta_{0}$. Since $\nu_{0}$ is outer regular, this yields a cover of $Y$ by open sets $G_{m}, 1 \leq m \leq n$, with $\nu_{0}\left(G_{m}\right)<\delta_{0}$. Now in a metrizable space each open set $G$ can be approximated from inside by a closed set $F$ with measure zero boundary $\partial F$ (choose a metric $d$ and consider the closed sets $F_{t}:=\{y: d(y, z) \geq t$ for $z \notin G\}$, having disjoint boundaries for different values $t>0$ ). Thus there are closed sets $F_{m} \subset G_{m}$ with

$$
\nu_{0}\left(F_{m}\right)>\nu_{0}\left(G_{m}\right)-\frac{1}{n} \delta_{0} \quad \text { and } \quad \nu_{0}\left(\partial F_{m}\right)=0 .
$$

Adjoining to $F_{1}, \ldots, F_{n}$ the set

$$
F_{0}:=Y \backslash \bigcup_{1 \leq m \leq n}\left(F_{m} \backslash \partial F_{m}\right)
$$

yields a cover of $Y$ by closed sets $F_{m}, 0 \leq m \leq n$, with $\nu_{0}\left(F_{m}\right)<\delta_{0}$ for all $m$, because also

$$
\begin{aligned}
\nu_{0}\left(F_{0}\right) & =\nu_{0}\left(\bigcup_{1 \leq m \leq n} G_{m}\right)-\nu_{0}\left(\bigcup_{1 \leq m \leq n} F_{m}\right) \\
& \leq \sum_{1 \leq m \leq n} \nu_{0}\left(G_{m} \backslash F_{m}\right)<\delta_{0} .
\end{aligned}
$$

Thus the weakly open set

$$
M_{0}:=\bigcap_{0 \leq m \leq n}\left\{\nu \in M(Y): \nu\left(F_{m}\right)<\delta_{0}\right\}
$$

contains $\nu_{0}$ and satisfies $\delta(\nu)<\delta_{0}$ for all $\nu \in M_{0}$, as had to be shown.

The final auxiliary result is the essential content of [, Lemma 4.1] (for a similar argument see the proof of [3, Lemma 1]):

Lemma 3. If $Y$ is a Hausdorff topological vector space, then for any $\nu \in M(Y)$ there are only countably many one-dimensional affine subspaces $S$ of $Y$ satisfying the inequality $\nu(S)>\delta(\nu)$.

Proof. Let $\mathfrak{S}$ denote the class of all one-dimensional affine (closed, hence Borel measurable) subspaces $S$ of $Y$ and $A$ be the set of points $y \in Y$ with $\nu(\{y\})>0$. Let, moreover, the measure $\nu_{0}$ be defined by $d \nu_{0}=1_{X \backslash A} d \nu$. Then $S \in \mathfrak{S}$ satisfies $\nu(S) \leq \delta(\nu)$, whenever (a) $S$ intersects $A$ in at most one point and (b) $S$ is a $\nu_{0}$-null set. But there are only countably many exceptions of (a), because $A$ is countable, and this holds as well for (b), because two sets $S \in \mathfrak{S}$ meet in at most one point and thus are $\nu_{0}$-almost disjoint.

\section{MAIN RESULTS}

No further auxiliary results on nonatomic measures (as in Section 3 of [6]) are needed to establish the following extension of Wulbert's main result:

Proposition 1. Let $\mu$ be a nonatomic $\sigma$-finite Baire measure on some topological space $X$ and define the topology in $\mathcal{C}(X)$ by the (finite or infinite) uniform norm. Then

$$
\mathcal{C}_{0}:=\{h \in \mathcal{C}(X): \mu(\{x: h(x)=y\})=0 \text { for all } y \in \mathbf{R}\}
$$

is a dense subset of $\mathcal{C}(X)$. 
Proof. 1. Since $\mu$ may be replaced by any dominating measure, it is sufficient to consider the case of a probability measure. Since, moreover, $\mu$ is nonatomic, there exists a Baire measurable function $h: X \rightarrow[0,1]$ with

$$
\mu(\{x: h(x)=y\})=0 \quad \text { for } 0 \leq y \leq 1 .
$$

Let $h_{n} \in \mathcal{C}(X), n \in \mathbf{N}$, be an $L_{1}$-approximation of $h$, i.e. satisfying

$$
\lim _{n \rightarrow \infty} \int_{X}\left|h_{n}-h\right| d \mu=0
$$

where $0 \leq h_{n} \leq 1$ may and will be assumed. Then $h_{n} \rightarrow h$ in $\mu$-measure and thus the distributions $\mu \circ h_{n}^{-1}$ converge weakly to $\mu \circ h^{-1}$. Since this distribution is nonatomic, Lemma 2 yields

$$
\limsup _{n \rightarrow \infty} \delta\left(\mu \circ h_{n}^{-1}\right) \leq \delta\left(\mu \circ h^{-1}\right)=0 .
$$

2. Now, with $f \in \mathcal{C}(X)$ and $n \in \mathbf{N}$ being fixed, define

$$
g_{t}:=f+t h_{n} \in \mathcal{C}(X) \quad \text { for } t \in \mathbf{R} .
$$

Then an application of Lemma 3 to $Y=\mathbf{R}^{2}$ and the joint distribution $\nu$ of $\left(f, h_{n}\right)$ shows the inequality $\delta\left(\mu \circ g_{t}^{-1}\right) \leq \delta(\nu)$ to hold, except for countably many values of $t$. Combined with the trivial inequality $\delta(\nu) \leq \delta\left(\mu \circ h_{n}^{-1}\right)$ this provides a dense subset $T$ of $\mathbf{R}$ such that

$$
\delta\left(\mu \circ g_{t}^{-1}\right) \leq \delta\left(\mu \circ h_{n}^{-1}\right) \quad \text { for } t \in T .
$$

3. Next, consider the sets

$$
\mathcal{G}_{n}:=\left\{g \in \mathcal{C}(X): \delta\left(\mu \circ g^{-1}\right)<\delta\left(\mu \circ h_{n}^{-1}\right)+\frac{1}{n}\right\} \quad \text { for } n \in \mathbf{N} .
$$

Since the mapping $\mathcal{C}(X) \ni g \mapsto \mu \circ g^{-1} \in M(\mathbf{R})$ is continuous, they are open subsets of $\mathcal{C}(X)$ by Lemma 2 . Since, moreover, they are dense in $\mathcal{C}(X)$ by part 2 of the proof and $\mathcal{C}(X)$ is a Baire space, the set $\bigcap_{n \in \mathbf{N}} \mathcal{G}_{n}$ is as well dense in $\mathcal{C}(X)$. Since, finally, this intersection equals $\mathcal{C}_{0}$ by part 1 of the proof, the assertion is established $*$

Now the concluding criterion is an easy consequence:

Proposition 2. A Baire measure $\mu$ on a topological space $X$ allows a continuous transformation $\varphi$ into Lebesgue measure on the interval $[0, \mu(X)]$ resp. $\mathbf{R}_{+}$if and only if it is nonatomic and moderated.

Proof. The condition is necessary, as is clear for "nonatomic" and follows for "moderated" from Lemma 1. To prove sufficiency choose a function $f \in \mathcal{C}(X)$ according to Lemma 1 and a function $h \in \mathcal{C}_{0}$ with $\left\|h-\left(\frac{1}{f}+1\right)\right\|<1$ according to Proposition 1. Then $h \geq 0$, and it follows from

$$
\mu(\{x: h(x) \leq y\}) \leq \mu\left(\left\{x: f(x) \geq \frac{1}{y}\right\}\right)<\infty \quad \text { for } y>0,
$$

that the "distribution function"

$$
\psi(y):=\mu(\{x: h(x) \leq y\}) \quad \text { for } y \geq 0
$$

\footnotetext{
* The original proof of Proposition 1 used arguments from measure theory, resulting in

$$
\delta\left(\mu \circ\left(f+\sum_{n \in \mathbf{N}} \frac{t_{n}}{2^{n}} h_{n}\right)^{-1}\right)=0 \quad \text { for } \quad \lambda^{\mathbf{N}} \text {-almost all }\left(t_{n}, n \in \mathbf{N}\right) \in[0,1]^{\mathbf{N}} .
$$
}

Following a suggestion by the referee, it was slightly simplified by using topological arguments. 
is continuous and finite-valued. Using the "inverse function"

$$
\psi^{-1}(z):=\max \{y: \psi(y) \leq z\} \quad \text { for } 0 \leq z<\mu(X),
$$

it is now easily checked that

$$
\mu(\{x: \psi \circ h(x) \leq y\})=y \quad \text { for } 0 \leq y<\mu(X),
$$

i.e. the continuous function $\varphi:=\psi \circ h$ is appropriate.

Finally, it should be mentioned that for infinite $\mu$ a continuous transformation into Lebesgue measure on the whole real line is as well possible. Indeed, to replace $X=\mathbf{R}_{+}$by $Y=\mathbf{R}$, it suffices to choose for the first step a continuous function $h$ with the properties

$$
\lambda(\{x:|h(x)|=y\})=0 \quad \text { and } \quad \lambda(\{x:|h(x)| \leq y\})<\infty \quad \text { for all } y \geq 0,
$$

satisfying in addition

$$
\lambda(\{x: h(x) \leq 0\})=\infty=\lambda(\{x: h(x) \geq 0\})
$$

(take for instance $h(x)=x^{2} \sin x$ ). The second step then uses the "two-sided" distribution function $\psi$, being defined by $\lambda(\{x: 0 \leq h(x) \leq y\})$ for $y \geq 0$ and by $-\lambda(\{x: y \leq h(x) \leq 0\})$ for $y \leq 0$, respectively.

\section{ADDED IN PROOF}

As the referee asked for a direct solution, here is a continuous transformation $\varphi$ of Lebesgue measure on $\mathbf{R}_{+}$into Lebesgue measure on $\mathbf{R}$ : define mappings $\varphi_{n}:[0,1] \mapsto \mathbf{R}_{+}, n \in \mathbf{N}$, by affine interpolation of the points

$$
(0,0),\left(\frac{1}{2(n+1)}, n\right),\left(\frac{1}{2}, n+1\right),\left(1-\frac{1}{2(n+1)}, n\right),(1,0)
$$

and let $\varphi$ be composed of the translates of $\varphi_{n}$ resp. $-\varphi_{n}$ to the intervals [ $2 n-$ $2,2 n-1]$ resp. $[2 n-1,2 n]$.

\section{REFERENCES}

1. Hobby, C.R. and J.R. Rice, A moment problem in $L_{1}$-approximation, Proc. Amer. Math. Soc. 16(1965)665-670. MR 31:2550

2. Kellerer, H.G., Zur Existenz analoger Bereiche, Z. Wahrscheinlichkeitstheorie Verw. Geb. 1(1963)240-246. MR 28:182

3. Kellerer, H.G., A topological version of Liapunov's theorem, Arch. Math. 72(1999) 206-213. MR 2000c:28019

4. Phelps, R.R., Uniqueness of Hahn-Banach extensions and unique best approximation, Trans. Amer. Math. Soc. 95(1960)238-255. MR 22:3964

5. Wulbert, D.E., Liapunov's and related theorems, Proc. Amer. Math. Soc. 108(1990) 955-960. MR 90m:46074

6. Wulbert, D.E., Annihilating a subspace of $L_{1}$ with the sign of a continuous function, Proc. Amer. Math. Soc. 128(2000)2431-2438. MR 2000m:46036

Mathematisches Institut der Universitaet Muenchen, Theresienstrasse 39, D-80333 Munich, Germany

E-mail address: hans_g.kellerer@mathematik.uni-muenchen.de 